\title{
A.I. Granovitch \\ Parasitic systems and the structure of parasite populations
}

Received: 15 January 1998 / Accepted: 15 October 1998

\begin{abstract}
The analysis of population systems is carried out on the basis of the spatial and functional classification of populations developed by V.N. Beklemishev. The population system is a functional part of a particular community. Steady interrelationships between population systems of different species within the community (referred to as "community links") appear to be a prerequisite for the formation of a complex of population systems. A prominent example of this is the parasitic system. The parasitic system is the population system of a parasite with all the connected populations of its hosts. The complexity of a parasitic system depends on: (1) peculiarities of the life cycle of the parasite, since its population system is the organizing component of the parasitic system and (2) subdivision of the environment for the parasites. The first trait is discussed from the standpoint of the phase structure of populations, which is clearly seen in parasites. The second one comprises the organization of the parasites' environment according to the scale of variability (interspecies, interpopulation or intrapopulation) of hosts. These make it possible to recognize spatial and functional parts in the framework of the parasitic system. A critical review of the terminology is presented together with a list of the pertaining vocabulary.
\end{abstract}

Key words Parasitic systems · Parasite populations . Population structure $\cdot$ Hemipopulations

\section{Introduction}

Considerable advances have been made during recent decades in the investigation of the ecology of parasitic organisms and their hosts. Moreover, there are also data on the genetic structure of parasite populations (Price 1980; Day et al. 1992; Truc and Tibayrenc 1993; Minch-

A.I. Granovitch

Department of Invertebrate Zoology, St. Petersburg State University, Universitetskaya nab. 7/9, 199034, St. Petersburg, Russia e-mail: Andrey@AG2304.spb.edu ella et al. 1995). At the same time, the general theories on the populations of parasitic organisms as well as the terminology used for descriptions are still far from being exact. This situation in my view reflects first of all a lack of understanding of the populations (both free-living and parasitic organisms) as holistic supraorganismic systems. Secondly, complicated spatiotemporal structures disguise the general features that characterize populations of any parasitic organisms.

This paper describes general peculiarities of the structure of populations of parasitic organisms. The main approaches, which are evolved here, were formulated by V.N. Beklemishev in his papers (Beklemishev 1956, 1959, 1960, 1964). These notions and the pertaining terminology apparatus are used more and more extensively by parasitologists and ecologists in Russia, but they are as yet unknown abroad.

Parasites are considered by Beklemishev as normal members of communities. Accordingly, a general conceptual apparatus was proposed for the description of the populations of parasitic as well as free-living organisms. Thus, we will first dwell briefly on the general concept of populations as supra-organismic systems and then more carefully on the peculiarities of parasite populations.

The analysis of any supra-organismic system is confronted with difficulties owing to the following features of these systems: the great diversity, slightly developed integrity, very complicated structure, low degree of organization and usually a low level of morphogenetic distinctness (Beklemishev 1964). This results in the weak morphological separation of the functional units of these systems. We should say that the most important morphological criterion for investigations on the organismic and suborganismic levels is only slightly acceptable for the analysis of supra-organismic systems including populations. It hinders the implication of a typological approach to the studies of these systems. The use of morphological criteria seems to have low efficiency for the elaboration of the typology of populations and intrapopulation groups. 


\section{Morphofunctional structure of the population systems of free-living and parasitic organisms}

The functional approach to the study of field populations implies that self-reproduction ${ }^{1}$ is the main feature of an independent population. "The greater the exchange of individuals is between two populations, the smaller their functional separation is" (Beklemishev 1960, cited after Beklemishev 1970, p. 233).

The functioning of population groups on different scales in the communities involves such effects as the greater or lesser exchange by migrants; the fusing of groups during an increase in population density, and the obviously strong isolation during periods of decreased population density; the formation and disappearance of temporal populations. These events occur within the limits of the functional complex of populations. The latter notion is defined by V.N. Beklemishev as a complex of groups comprised of one or more independent populations together with various dependent groups supported by the independent populations, as well as by several independent populations connected by extensive migration (Beklemishev 1960). ${ }^{2}$

It is worth noting that the functional complex of population has certain spatial features. This is a system of groups that are "...in particular spatial association" (Beklemishev 1960, cited after Beklemishev 1970, p. 237). Therefore, a recognition of these complexes allows us to evolve a morphofunctional approach to the analysis of the field populations. Within the frame of this approach, intensive case studies have been made on the field populations of some fish species (Altukhov 1983), insects (Kreslavsky et al. 1976, 1987), etc. The ecological-genetic analysis of the systems of subpopulation units leads to the important conclusion: the characteristics of the entire system are much more stable than the respective parameters of the subpopulation groups in which a great variability is observed. Thus, the population system is a

\footnotetext{
That is, reproduction of population due to reproduction of its individuals, but not due to immigration of individuals from neighbouring populations. According to this criterion, several groups of individuals could be distinguished. They differ in the balance between self-reproduction and migration rates. If self-reproduction prevails over immigration rate, the population is independent. Otherwise, if survival of the population is maintained mainly by immigration, then, in this case, we are dealing with a pseudopopulation. On the basis of these differences in survival, depending on the success or failure of self-reproduction, a whole range could be found, from permanent populations to temporal or periodically appearing populations, including micropopulations (Beklemishev 1960).

2 Such complexes of populations were later designated as metapopulations. The prefix "meta" etymologically means sequence (here - sequence of populations) and was first proposed to describe the system of local populations (Hanski and Gilpin 1991). Some of these become extinct and are then re-established by individuals immigrating from other local populations. At the same time, a metapopulation as a whole (but not its parts) is considered to be quite stable in a time system. This is why I prefer to use the more accurate term "functional complex of populations", an earlier proposal, or the term "population system".
}

stable unit which is comprised of the smaller groups that are isolated to a different extent. The specific features of this stable unit could not be defined by the simple summation of the features of its separate parts. Population systems can be considered as the functional complexes of populations. Population systems may consist of various groups. These groups may differ in the ability to self-reproduce and the degree of spatial isolation. This is one aspect of the morphofunctional structure of the population system.

A morphofunctional approach to the analysis of the structure of field populations allows us to emphasize another aspect, namely, the existence of certain parts of the population systems which are "specialized" to different extents. These parts (phase groups - see below) have specific functions in the whole population system. These groups could be recognized in accordance with the structure of a particular life cycle. As a rule, such groups are spatially separated and have specific ecological features. The most prominent differences between these groups are characteristic of the so-called heterotopic species (Beklemishev 1960).

In general, populations, as elements of communities, are hierarchical systems with an inherent complicated functional structure. In order to stress this, the term "population system" seems to be appropriate. It meansm on the one hand, that these systems are composed of the lower hierarchical groups with different degrees of selfreproduction, and, on the other, that they comprise different functional groups according to the life cycle stages (phase features). These two aspects of the structure of the population systems reflect the differences between para- and metagroups. Those elements of the population systems that are functionally similar, i.e. "parallel", we could define as the paragroups (parapopulation groups). So, despite the different degree of self-reproduction, the more or less isolated populations, which together enable the functioning of the population system, form the parapopulation row. As far as the second aspect of the structure of the population systems is concerned, all the "successive" groups, with respect to the life cycle, form the metapopulation row which is a system of metapopulation groups that regularly interchange during the life cycle. The difficulties of the morphofunctional analysis of field population systems appear all the more evident when populations of parasites are considered. This is due to: (1) the difficulties in recognizing population boundaries; (2) the two-faced nature of the environment which is composed of the external environment (the secondary environment) and the organism of the host (the primary environment ) (Pavlovsky 1934). It is necessary to keep in mind that host populations have, in turn, a complicated spatiotemporal organization; (3) the specific features of the life cycles of the parasites covering a sequence of morphologically distinct stages. The peculiarities of the parasite populations (in contrast to free-living organisms) may seem so significant that they have resulted in the creation of a special nomenclature of the subpopulation groups (Macko 1979; Romashov 1990). Thus, a cat- 
egory of subpopulation groups (intrapopulations, extrapopulations, different types of phenotes, etc.) was evolved on the basis of very particular and formal criteria. According to this category, these groups cannot be related either to groups of similar scale in free-living animals, or to subpopulation groups in other parasites with a differing life cycle. Moreover, in this classification the functional value of the given subpopulation group for the entire population system remains unclear.

A consideration of the organization of the parasite populations as a special case of the population systems of any organism appears to be the more promising approach. Moreover, the subdivision of the environment of the parasites could be a model which makes it possible to gain a more adequate estimation of the structure of the population systems of free-living organisms. For instance, a comparison of the population systems of parasites and free-living organisms could prove useful if it reveals elements of equal functional value and, consequently, this should help us to form a general view of the population systems.

\section{Community connections and parasitic systems}

Every population system is part of a certain community. In a community, the population system interacts with the population systems of other species. These interactions can be more or less close and regular. In the case of stable and regular interactions between individuals of two species, the stable community connection arises between the population systems of these species. The most striking examples of community connections (in other words, the complexes of the greatest system integrity which could be easily detected in the community) could be seen in obligatory mutualistic interactions of individuals. In this case, the connection of the population systems is characterized by a high level of integration. The stable complexes of the population systems are formed also by other types of connections between individuals, i.e. competition, predator-prey interactions, etc. In general, the more important the organism of one species is for the organism of another, the greater the entirety of the complex that makes up their population systems.

Population systems of the parasitic organisms in the community are in contact with the population systems of other species: firstly, of their hosts, but also of predators, competitors and so on. The interactions of the individual hosts and parasites are very close and include a complex of the topic, trophic interactions allowing us to define a host organism as the environment for the parasite (Pavlovsky 1934). At the population level, these interactions are reflected in the stable existence of the complex of several population systems. This complex involves a population system of the parasite and the population systems of all the host species connected with this parasite. A complex of the population systems of hosts united by the population system of a parasite was designated as a parasitic system (Beklemishev 1956). Numerous investi-

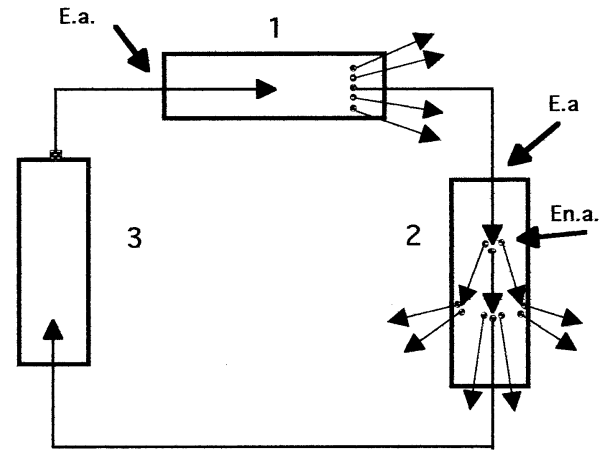

Fig. 1 Schematic representation of a trixenic life cycle of trematodes, giving an example of a complex cycle that results in establishing a parasitic system with at least three host populations. 1 Definitive host; 2 first intermediate host; 3 second intermediate host; E.a. exogeneous accumulation; En.a. endogeneous agglomeration

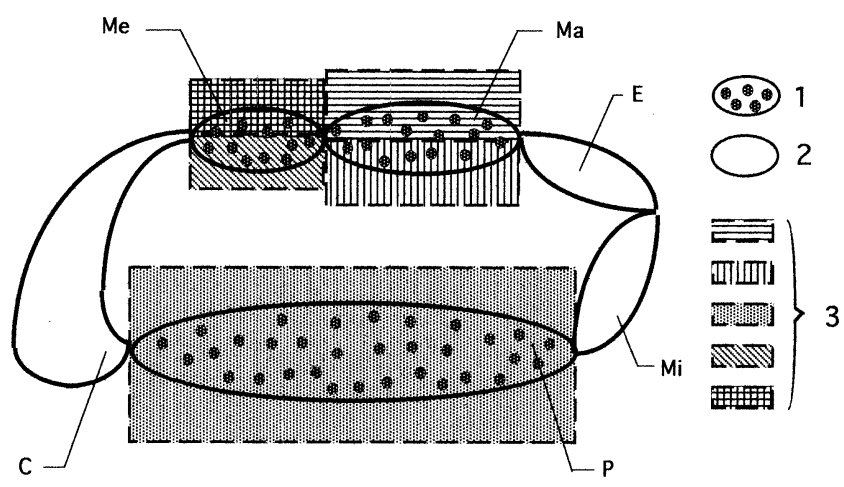

Fig. 2 Parasitic system formed by population system of the trematode with a trixenic life cycle. 1 Hemipopulations of parasitic stages; 2 hemipopulations of free-living stages of parasite; 3 population systems of different host species. Hemipopulations: Ma marites; $E$ eggs; $M i$ miracidiae; $P$ parthenogenetic generations (parthenites); $C$ cercariae; $M e$ metacercariae

gations devoted to the field populations of parasites reveal a high level of integrity of the parasitic system. In many cases, the existence of feedback mechanisms has been shown (Kennedy 1978; Esch and Fernandez 1993). Moreover, the interactions of individuals (= elements of the parasitic systems) are very integrated: evidence reveals the importance of molecular and genetic interactions between parasites and their hosts (Wakelin 1978; Soprunov 1987).

A minimum of two population systems is required for a parasitic system - a population system of the parasite and that of the host. However, this is a rare situation in nature that appears only in the case of a monoxenic life cycle and strict specificity of a parasite. As a rule, a parasitic system consists of more elements (= population systems): parasites of a single developmental stage may use several populations of the different host species which in this case are termed paraxenic hosts. Additional extension of the number of interacting populations occurs if the parasite has a di-, tri or tetraxenic, etc. life cycle (Figs. 1,2). 


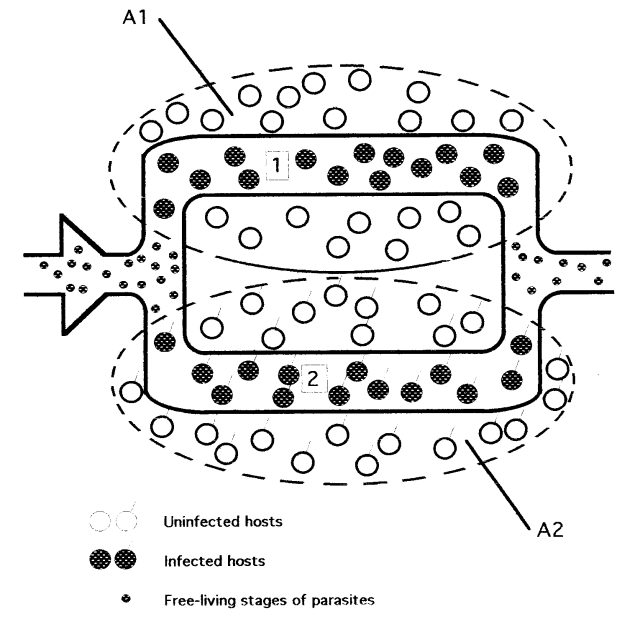

Fig. 3 Schematic representation of a part of the parasitic system. Interactions of the parasite hemipopulation with two population systems $(A 1$ and $A 2)$ of the same host species. 1, 2 Parts of the hemipopulation of a parasite

It is worth noting that the population system of the parasite in the community not only interacts with the host population systems; the parasites may form stable community connections with the population systems of other animals, for example, of some non-host animals feeding on the dispersal stages of parasites. These connections are based on predator-prey relationships and could be important for the community (Shigin 1978). Thus, we can extend the concept of a parasitic system. We should consider the whole range of population systems of all the organisms which form the community connections of any type with the studied population system of the parasite. However, in this paper we will keep to a traditional meaning of the term "parasitic system" which involves only the host-parasite connections in the community.

The boundaries of the parasitic systems are believed to lie within the community (Beklemishev 1956). Actually, a whole necessary set of population systems is available, providing the parasite circulation within a single community. At the same time, it is obvious that hosts capable of wide spatial distribution could provide a wider dispersal of parasites. Thus, parasites from the same parasitic system (single population system of parasites) may circulate in several, and sometimes spatially separated, communities. Thus, it must be taken into account that the single parasitic system may comprise a number of independent population systems of the same host species. For example, several spatially separated population systems of the mollusc, which is a first intermediate host for trematodes, are united into a parasitic system by the migration activity of the vertebrate definitive hosts (see Fig. 3).

It is also necessary to emphasize another feature of the parasitic system, namely, the role of this system as a link between different biocoenoses. This role is connected with the heterotopic character of the life cycle of the parasites. As a result, the population systems of organisms belonging to different biocoenoses are integrated into a complex system. For example, populations of planktonic crustaceans, as first intermediate hosts of many pseudophyllid cestodes, and population systems of fishes, as their second intermediate hosts in the trixenic life cycle, represent biocoenoses of lake or river. They comprise the same parasitic system as populations of some vertebrate fish-eating animals as final hosts of these cestodes. The latter population can belong to another biocoenosis, for example a terrestrial one, such as a forest.

The structure of parasitic systems is very variable; there is, however, a common basis of their organization. The structure of these complicated systems is generally determined by: (1) the specific life cycle of the parasites, the population system of which is an organizing component of the parasitic system; (2) the subdivision of the environment of the parasites. The latter is a result of the mosaic nature of the environment in the case of the freeliving stages, and of the ability to exploit the hosts' heterogeneity in the case of the parasitic stages, including the populations of the different host species, and interand intrapopulation heterogeneity of the host individuals. Later on, I will concentrate on the general aspects of the organization of the parasitic systems and the population systems of parasites.

\section{Phase structure of the population systems: hemipopulations}

With regard to the different structural aspects of the parasite population systems (and, respectively, the complex organization of the parasitic system), it is necessary to stress that the primary subdivision of the populations on the basis of the life cycle structure is typical for any living organisms. An organism which has a simple life cycle passes through several developmental stages (=phases) in its ontogeny. If these stages are clearly morphologically and physiologically separated and connected by the periods of the fast morphological rearrangements (e.g. metamorphosis or hatching of larvae from the eggs), the different groups of individuals will occur simultaneously or consecutively in the population. The greater the morphophysiological differences between the stages of the life cycle and the more discrete their characteristics, the greater the ecological separation of the parts of the population system comprising these stages. In this case we can call this a prominent phase structure; that is, the phase structure of the population system could be defined as follows: a composition of the population systems from the subpopulation groups which consist of organisms with similar morphophysiological peculiarities typical of the different stages of the life cycle.

Insofar as the phase structure of the population system is connected with the peculiarities of the life cycle and with the ontogeny of the organisms, it reflects a specific age structure. However, we do not mean an "ab- 
solute" (astronomic) age, but the physiological stage of the ontogeny, i.e. "physiological" age. It becomes obvious when we find organisms of different generations belonging to the same phase group, e.g. the phase group of imago of the May beetle (Melolontha hyppocastani) is not uniform with respect to age. It includes individuals that have spent different periods (number of years) at the larval stage (Yablokov 1987). At the same time, the imago group comprises an entity regarding the morphophysiological and ecological features of the individuals. It provides a good example of a clearly outlined phase group.

The most pronounced examples of the phase structure can be found in the population systems of organisms undergoing metamorphosis during the life cycle. In this case, the separation of the population system into two or more phase groups means the "specialization" of these parts of the population systems. Trophic and reproductive groups could be distinguished. The group of the resting stages is a quite unique one. ${ }^{3}$ In extreme cases, the specialization of parts of the population system may be practically complete (e.g. in the orders Ephemeroptera and Trichoptera, and in marine invertebrates with lecitotrophic larvae). This considerable level of specialization is connected with the prominent spatial subdivision in the population system: different phase groups may belong to different ecosystems. At the same time, they are parts of a single population system.

A more complicated phase structure of the population system is observed in the case of altering generations (a complex life cycle). Individuals of each generation can form one or several phase groups. By contrast, the least pronounced phase structure is typical of the population systems of those organisms which have a simple life cycle, the lack of metamorphosis, and viviparity (ovoviviparity) as the only mode of reproduction. In this case, the phase structure becomes "blurred". This is expressed in the relatively weak differences between different age groups. Phase structure is practically absent in the population systems of some amictic organisms.

Therefore, in the case of prominent phase structure, the subpopulation groups that are more or less specialized could be easily identified. To define such subpopulation groups, the term "hemipopulation" was proposed by V.N. Beklemishev (Beklemishev 1960). Correspondingly, the subdivision of the population system into several hemipopulations reflects the phase structure of this system. The hemipopulations, in turn, are functional parts of the population system.

Phase structure (meaning the occurrence of easily detectable hemipopulations) is, to a greater or lesser extent,

\footnotetext{
3 The problems of the functional significance of the phase groups for the population system are still poorly understood and need a separate discussion. So, it is obvious that the subpopulation groups of "resting stages" may include organisms enduring an active morphogenetic rearrangement. At the same time, we can define those individuals as "resting" which persist under unfavourable environmental conditions. In this case, the organisms are characterized by "physiological rest".
}

characteristic of the free-living organisms. At the same time, this feature is always extremely inherent in the population systems of parasites. Actually, the strong subdivision of the environment, combined with the complicated life cycle of parasites, inevitably leads to the formation of different subpopulation groups. Among them, hemipopulations of free-living stages (hemipopulations of eggs in the environment, hemipopulations of freeswimming larvae, etc.) can be recognized. Hemipopulations of the parasitic stages are ecologically contrasted with the above-mentioned ones. Moreover, parasites frequently have complex life cycles. Hence, this leads to a further increase in the number of subpopulation groups and to an even higher complication of the phase structure of the population system. For example, in the case of the trixenic life cycle of trematodes we find hemipopulations of larval stages, those of marites, parthenogenetic generations, and metacercariae. These are parts (and quite specialized parts) of the whole population system of the parasite. As in the case of free-living animals, we can see that the most prominent phase structure of the population system corresponds to the specialization of the parts (separate hemipopulations) of this system. Hemipopulations of the non-feeding stages are considered to have a function of spatial and/or temporal dispersal. To be more precise, they provide integration of the whole system. Successive hemipopulations accomplish a trophic or reproductive function or both.

Naturally, the phase structure of the population system of parasites and the composition of this system of several hemipopulations is important for the organization of the parasitic system. The population systems of a certain host species interact with the hemipopulations of the parasite. In this case, a functional specialization of the separate hemipopulations of parasites corresponds to the functional specialization of the parts (blocks) of the parasitic system, each of which is composed of the hemipopulation of a parasite and the population system of a respective host. (Examination of the structure of the parasitic system from the viewpoint of the functional specialization of its parts appears to be very promising and important. This question needs further consideration.)

\section{Environmental subdivision and the structure of the hemipopulation of parasites}

Analysis of the structure of the parasitic system (and the population system of parasites as an organizing component) cannot be restricted only to considerations of the phase structure and the corresponding set of hemipopulations. Firstly, it is necessary to note that the single hemipopulation of the parasite (parasitic phase) is subdivided into several parts corresponding to the different population systems of the same host species (see an example of the trematodes given above; Fig. 3). Thus, the interpopulation component of the host's diversity determines the specific features of the environment for different parts of the same hemipopulation of the parasite. 
The differences between population systems of the same host species can be appreciable. Different features of the host's population structure (e.g. age structure, sex ratio, other peculiarities of the reproductive structure as well as temporal changes of these characteristics), which are of importance for the parasite, may vary in different populations of the host (Granovitch and Sergievsky 1990; Sergievsky et al. 1991). It seems important that different population systems of the same host species are genetically independent and differentiated. A consistently growing body of data suggests the existence of genetic heterogeneity of the host populations, including variation in traits related to susceptibility or resistance to parasites (Wakelin 1978; Munger et al. 1986; Wassom et al. 1989).

The estimation of the significance of the above-described subdivision for the functioning of the population system of a parasite and the parasitic system as a whole is a subject for future investigations. At present, it should be emphasized that there is no developed term for the parts of the parasite's hemipopulation connected with the different population systems of the same host species.

The second peculiarity of the hemipopulations of the majority of parasites is the subdivision according to the population systems of different host species (Fig. 4). This feature (so-called paraxenia) is connected with the degree of specificity of the parasite. In fact, the individuals of several host species may further the survival, growth and reproduction of the parasites of the same phase group. In this case the hemipopulation of the parasite interacts with the several population systems of paraxenic hosts.

Thus, the heterogeneity of the parasites' environment is determined by the interspecific differences of the hosts. Each "paraxenic" part of the parasite hemipopulation faces different environments. The specific features of this environment are dependent on the morphophysiological and ecological differences of the host individuals of different species, as well as on the interspecific differences in an organization of the hosts' populations. The investigation of the specificity of the parasites has shown the prominent interspecific inequality of the host individuals for parasites. The species-specific characteristics of the host influence essential traits of the parasites, such as mortality, reproduction intensity and time of development. At the population level of interactions, specificity is determined, on the one hand, by the specificity of individual interaction and, on the other hand, by the peculiarities of the structure and dynamics of the host population. In general, it is necessary to elaborate a new concept analogous to the specificity, but at the population level. Appropriate estimates made on the basis of this concept must define the parameters of the part of the parasite's hemipopulation connected with a certain host population and compare them with the respective parameters of the analogous parts connected with the populations of other hosts. From the viewpoint of the parasitic system, this will mean obtaining the characteristics of the parts of the ramified "flow" of parasites which are supported by the population systems of different host species (see Fig. 4).

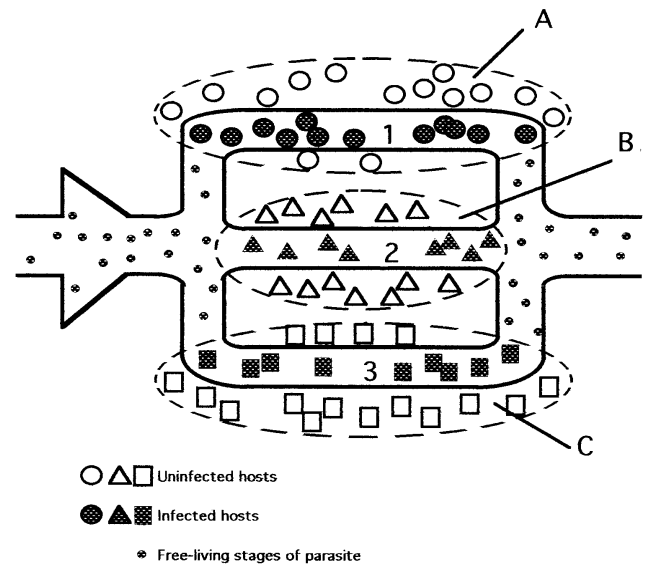

Fig. 4 Schematic representation of a part of the parasitic system. Interaction between the parasite hemipopulation and the population systems of three host species $(A, B, C) .1,2,3$ Parahemipopulations of parasite

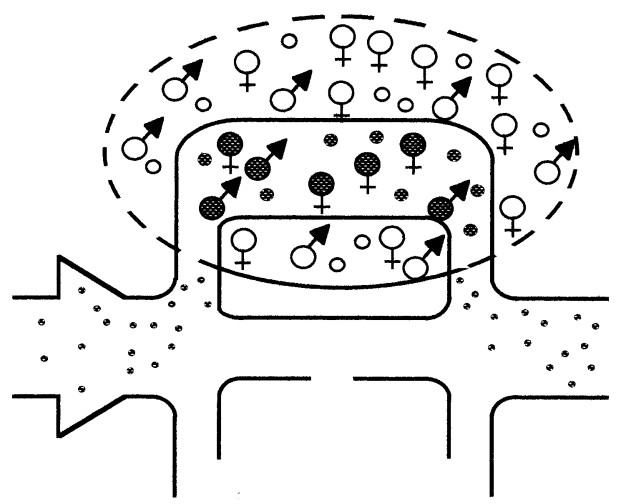

Fig. 5 Schematic representation of a part of the parasitic system. Interactions between one part of a parasite hemipopulation (parahemipopulation) and the population system of the host. Note the heterogeneity of host individuals in gender and age which results in the heterogeneity of the environment for the local hemipopulations of a parasite. Smallest circles represent free-living stages of parasites

Further investigations into the subdivision of the population system of parasites lead to consideration of even smaller groups within the hemipopulation and inside the separate parts of the hemipopulation connected with paraxenic hosts. These smaller groups consist of the aggregations of conspecific parasite individuals harboured by a certain host individual. Such groups are traditionally defined in Russian scientific literature as local hemipopulations (Galaktionov and Dobrovolsky 1984). This term emphasizes that these groups are parts of a hemipopulation. Indeed, all the local hemipopulations in assemblage form the whole hemipopulation.

Local hemipopulations are characterized by relatively short persistence which is shorter or equal to the longevity of the host individual. The environmental heterogeneity of local hemipopulations is dependent on the intrapopulation inequality of the host individuals (Fig. 5). There are numerous examples of the age-specific, sex- 
specific, etc. differences in the infection of the hosts (e.g. Dogiel 1963; Kennedy 1978). They represent an intrapopulation level of host heterogeneity and, consequently, an additional level of the environmental subdivision for the parasite populations. Moreover, striking intrapopulation differences between the host individuals with respect to susceptibility to parasites could be found. Hence, the "genetic component" of the heterogeneity of the parasites' environment should be stressed. The local hemipopulations of several parasites with complex life cycles acquire peculiar traits. These local hemipopulations are distinguished by their capability of self-reproduction within the host individual. Thus, these true local hemipopulations are at the same time micropopulations. The term "micro-hemipopulations" seems appropriate for the designation of such groups (Beklemishev 1959). The local hemipopulations of the parthenogenetic generations of some trematode species and the local hemipopulations of certain Mastigophora and Sporozoa are examples of such groups.

Prior to the summary of the analysis of the structure of the parasite population systems, it is necessary to clarify some terminological questions. In this paper, we do not intend to develop a special terminology for the description of the population systems of the parasites. Moreover, our aim is to show a necessity for a unified approach to the description of any population system either of parasites or free-living organisms. In our opinion, the terminology proposed by V.N. Beklemishev satisfies the latter requirement. However, even the superfluous examination of the population structure of parasites, as given above, necessitates a more differential approach to the investigation of intrapopulation groups. An earlier attempt to formalize the complicated relations of such groups has been made, whereby three "population levels" were suggested: supra-, meta-, and infrapopulations (see, for example, Esch and Fernandez 1993). This terminology has become official (Margolis et al. 1982) and is extensively applied in parasitology literature. So, it seems important to correlate this terminology with the terms used in the present paper. It is even more important because the problems of terminology frequently reflect marked differences in the approaches and the ideology of investigations.

The term "suprapopulation" was coined for the designation of all stages of the parasite present in the community (Esch et al. 1975). From the viewpoint of the parasitic system, this group should be defined as a population (more exactly, a population system) of parasite. It is worth noting that the notions of suprapopulation and population system are equal if all stages of the life cycle of the parasite circulate in the same community. The notion of population system is wider. It covers the groups of parasites belonging to different communities (e.g. integrated by means of the high mobility of the hosts) but functioning as an entire system.

In contrast to suprapopulation, the term "infrapopulation" defines the smallest groups of parasites and means a group harboured by a single host individual (Esch and
Fernandez 1993). The definition of infrapopulation completely coincides with the definition of local hemipopulation, but the term "local hemipopulation" seems to be much more universal. It could be applied to the groups of the particular scale of any organisms, both parasites and free-living organisms.

Among others, a distinction of metapopulations is important for the appreciation of the structure of the parasite population systems. The term "metapopulation" emphasizes the sequence of the functional groups in the population system (metapopulation row). (It is necessary to stress that "metapopulation" here has another sense than previously discussed (see above). It has nothing to do with a system of local populations which are interrelated by different levels of migrants.)

The metapopulation is defined as including all the parasites of a similar developmental stage connected with the individuals of a certain host species (Riggs et al. 1987). From this term it is clear that the authors mean the total of the infrapopulations made up by the same stage of the life cycle. It is important to notice that from the viewpoint of the parasitic system, this definition is in complete agreement with the earlier proposed term "hemipopulation".

At the same time, there is a profound inexactitude in the definition of a metapopulation. All the parasite individuals of the same stage but only a certain host species are mentioned. Accordingly, the groups of the parasite in other (paraxenic) host species are outside the consideration, although a paraxenia is a rule rather than an exception for the parasitic systems.

The groups of parasites in the paraxenic hosts mark the certain level of the environmental subdivision. This heterogeneity is connected with the interspecific differences of the host (see above for details). We are dealing with groups of parasites at the same life cycle stage maintained by different host species. For the population system of the parasite as a whole, this means a separation within the phase groups (hemipopulations). In this case, we must deal inevitably with the parts of the hemipopulations. It seems appropriate to give a more exact name for these parts. We suggest the term "parahemipopulation" in order to emphasize their place in the population system.

In general, the population system of parasites is comprised of groups of different scales and unequal functional significance. The smallest subpopulation groups - local hemipopulation - are connected with single host individuals. These are formed by an accumulation or an endogenous agglomeration (Dogiel 1963; Galaktionov and Dobrovolsky 1984). In most cases, these groups are not capable of self-reproduction. Local hemipopulations are combined to form parahemipopulations. The peculiarities of the environment for every parahemipopulation reflect the features of the different host species which support certain stages of the parasites. Moreover, different parahemipopulations of a parasite are connected with the different population systems of the same host species. The system of parahemipopulations, like the 
parallel "channels" of the united though ramified stream of parasites, is merged to form a hemipopulation. It is comprised of all the parasite individuals at a certain life cycle stage in whatever host is maintaining the parasite. In turn, a total of hemipopulations gives rise to a whole population system of the parasite. The number of hemipopulations is dependent on the structure of the life cycle of a parasite and reflects the phase structure of its population system.

Among hierarchically organized hemipopulations of the parasitic stages, the parasite usually has hemipopulations of free-living stages. The structure of the latter, in turn, may be considered from the viewpoint of its spatial differentiation and environmental heterogeneity. As is the case for any free-living organism, the extent of development of these structures depends on the patchiness of the environment and the local conditions in the different patches inhabited by the local hemipopulations. In general, we should emphasize that the hemipopulation structure of free-living organisms is in most cases not as easily discernible as in parasites. The identification of the subpopulation groups within the hemipopulation and the comparisons of these groups is dependent on the peculiarities of their microbiotopes. It is the discrete nature of the environment of the parasites and the organization of this environment with respect to the interspecific, interpopulation and intrapopulation variability of hosts which enable us to distinguish the spatially and functionally discrete parts of the hemipopulations in the parasitic system.

\section{Vocabulary}

The structure of the population systems

Population system - a complex of groups of conspecific individuals comprising one or more independent populations together with various dependent groups, supported by independent populations, as well as by several independent populations connected by extensive migration. Population system is a functional system of parapopulation and metapopulation groups (after Beklemishev 1960). Independent population - group of conspecific individuals, which multiply by local recruitment. Immigration is not necessary for the reproduction of this group (Beklemishev 1960).

Dependent population - group of conspecific individuals which partly reproduce by local recruitment. The level of recruitment here is not enough to compensate for mortality. The immigration of individuals from other populations is a necessary prerequisite for the survival of dependent populations (Beklemishev 1960).

Temporal population - group of conspecific individuals which occupy a suboptimal environment over a period of time (whereby the environmental conditions usually improve during this period). Temporal populations are capable of reproducing themselves by local recruitment during this period of time (Beklemishev 1960).
Pseudopopulation - group of conspecific individuals which are unable to replace themselves due to the fact that reproduction of individuals is absent or all individuals of the reproductive stage have been removed from the locality (for example by water currents) (Beklemishev 1960).

Micropopulation - independent populations in microbiotopes (live animals, dead animals, excrements of animals, burrows and nests of animals, live plants, dead trunks, small pools of water, epiphytic species, etc.). Micropopulations are able to replace themselves as long as their microbiotope exists (Beklemishev 1959).

Hemipopulation - group of conspecific individuals of one phase of life cycle, i.e. a group of individuals of similar morpho-physiological and ecological features. In the case of the prominent phase structure of population, different hemipopulations inhabit different biotopes (Beklemishev 1960).

Local hemipopulation - spatially isolated part of hemipopulation. Local hemipopulations are restricted to favourable patches of environment. A local hemipopulation of parasites is a group of conspecific parasites at the same stage of the life cycle within/on the host individual. As local hemipopulations comprise individuals of only one stage of the life cycle, they are not able to reproduce. All local hemipopulations together form a hemipopulation (Galaktionov and Dobrovolsky 1984).

Microhemipopulation - a special type of the local hemipopulation of some parasites with a complex life cycle. These local hemipopulations are distinguished by their capability of self-reproduction within/on the host individual (Beklemishev 1959).

Parahemipopulations - set of local hemipopulations connected with different types of habitat. For the parasites - a set of local hemipopulations which inhabit hosts of different species. Parahemipopulations play a similar role in the functioning of the population system as a whole.

Parapopulation groups - functionally similar parts of the population system.

Parapopulations - more or less isolated independent and dependent populations which jointly provide the functioning of the population system.

Parahemipopulations - see above.

Metapopulation groups - "successive" groups with respect to the life cycle, i.e. metapopulation groups comprise individuals at different stages of the life cycle. Different hemipopulations of the same population system are metapopulation groups. Metapopulation groups are significantly different with regard to function (for the population system as a whole) in the population systems with prominent phase structure.

Phase groups - set of conspecific individuals at the same stage of their life cycle. A phase group comprises individuals which are characterized by similar morphophysiological and ecological peculiarities. Phase groups are clearly visible if organisms pass through several morphologically and physiologically separated developmental stages connected by periods of fast morphophysiological rearrangements during their life cycle. 
Phase structure of population - composition of the population system which comprises phase groups (Galaktionov and Dobrovolsky 1984).

Life cycle, environment and community connections

Community connection - interrelations of population systems of two species in the community that are stablein-time due to stable and regular interactions between individuals of these two species (predator-prey, parasite-host, mutualistic, competition and other types of interindividual interactions) (Beklemishev 1956).

Parasitic system - complex of population systems of hosts, united by the population system of their parasite on the basis of stable parasite-host community connections (Beklemishev 1956).

Primary environment for the parasite - organism of the host (Pavlovsky 1934).

Secondary environment for the parasite - the external environment of the host (Pavlovsky 1934).

Complex life cycle - type of life cycle with two or more distinct, regularly alternating generations. These generations are usually characterized by different modes of reproduction.

Heterotopic species - species that inhabit different biotopes according to individuals at different stages and generations of their life cycle (for example terrestrial species with the water larvae, parasites and so on) (Beklemishev 1960).

Monoxenic life cycle - for the parasites - ability of the parasite to complete its full life cycle in/on the organism of only one host species.

Di-, tri-, tetraxenic life cycle - for the parasites - necessity of more than one host for the full completion of their life cycle; i.e. necessity of two metaxenic hosts for a dixenic life cycle, three metaxenic hosts for a trixenic life cycle and so on.

Homoxenic life cycle - for the parasites - ability of the parasite to use only one host species for every stage of its life cycle. This is the case of extremely strict specificity of parasite.

Heteroxenic life cycle - for the parasites - ability of the parasite to use more than one host species for some or all stages of its life cycle. A heteroxenic life cycle comprises several paraxenic hosts.

Paraxenia - ability of the parasite to use several species of hosts for the development of the same stage of its life cycle.

Paraxenic host - different species of hosts which are used by the parasite at the same stages of its life cycle. In this case strict specificity is absent.

Metaxenic host - different species of hosts, which are used by successive stages of the life cycle of the parasite. Accumulation - formation of the local hemipopulation through the repeated invasion of individuals into the microbiotope (for the parasites - into/on the host individual) (Dogiel 1963).

Endogenous agglomeration - formation of a local hemipopulation by the ingress of one individual into the microbiotope and the following reproduction of this individual (for the parasites - by penetration of the invasion stage into/on the host individual and several cycles of reproduction of this individual) (Dogiel 1963).

Parthenogenetic generations of trematodes - generations of sporocysts and rediae (Ginetsinskaya 1968).

Marites - individuals of hermaphroditic generations of trematodes, which parasitize the definitive host (Ginetsinskaya 1968).

Acknowledgments I am very grateful to Dr. A.A. Dobrovolsky for valuable advice, on numerous occasions, and attention during the preparation of the manuscript. Further thanks are due to Prof. Claude Combes and Prof. Kerstin Johannesson for their critical review and discussions of an earlier draft. I thank Dr. I Sokolova for assisting in the preparation of the text in the English language.

\section{References}

Altuchov UP (1983) Genetic processes in populations. Nauka, Moscow, 279 pp (in Russian)

Beklemishev VN (1956) Agents of illnesses as members of the community. Zool Zh 35:1765-1779 (in Russian)

Beklemishev VN (1959) Populations and micropopulations of the parasites and nidicoles. Zool Zh 38:1128-1137 (in Russian)

Beklemishev VN (1960) Spatial and functional structure of populations. Byull Mosk Obshch Ispyt Prir 65:41-45 (in Russian)

Beklemishev VN (1964) About the general features of the organization of living things. Byull Mosk Obshch Ispyt Prir 69:22-38 (in Russian)

Beklemishev VN (1970) Biocoenotic basis of comparative parasitology. Nauka, Moscow, 504 pp (in Russian)

Day KR, Koella JC, Nee S, Gupta S, Read AF (1992) Population genetics and dynamics of Plasmodium falciparum: an ecological view. Parasitology 104:S35-S52

Dogiel VA (1963) Allgemeine Parasitologie. Fischer, Jena, 523 pp

Esch GW, Fernandez JC (1993) A functional biology of parasitism. Chapman \& Hall, London, $337 \mathrm{pp}$

Esch GW, Gibbons JW, Bourque JE (1975) An analysis of the relationships between stress and parasitism. Am Midl Nat 93: 339-353

Galaktionov KV, Dobrovolsky AA (1984) Population analysis of the life cycles of trematodes on the basis of investigations of the microphallides of "pygmaeus" group (Trematoda: Microphallidae). In: Polyansky, YuI (ed) Ecological-parasitological investigations of the north seas. Academy of Sciences USSR, Apatity, pp 8-41 (in Russian)

Ginetsinskaya TA (1968) Trematodes - their life cycles, biology and evolution. Nauka, Leningrad, 411 pp (in Russian)

Granovitch AI, Sergievsky SO (1990) The appreciation of the reproductive structure of the populations of Littorina saxatilis (Olivi) (Gastropoda: Prosobranchia) in the White Sea. Zool Zh 69:32-41 (in Russian)

Hanski I, Gilpin M (1991) Metapopulation dynamics: brief history and conceptual domain. Biol J Linn Soc 42:3-16

Kennedy KR (1978) The ecological parasitology. Mir, Moscow, 230 pp (in Russian)

Kreslavsky AG, Micheev AV, Solomatin VM, Gricenko VV (1976) Intrapopulation ecological differentiation in the beetle Chrysochloa cacaliae. Zool Zh 55:1163-1171 (in Russian)

Kreslavsky AG, Micheev AV, Solomatin VM, Gricenko VV (1987) The exchange and ecological-genetic differentiation of the sympatric population system in Lochmaea capreae (Coleoptera, Chrysomellidae). Zool Zh 66:1045-1054 (in Russian)

Macko JK (1979) Population and classification of intrapopulation units of helminths. Folia Parasitol 26:201-219

Margolis L, Esch GW, Holmes JS, Kuris AM, Schad GA (1982) The use of ecological terms in parasitology (report of an ad 
hoc committee of the American Society of Parasitologists. J Parasitol 68:131-133

Minchella DJ, Sollenberger KM, Preira De Souza C (1995) Distribution of shistosome genetic diversity within intermediate hosts. Parasitology 111:217-220

Munger JC, Karasov WH, Chang D (1989) Host genetics as a cause of overdispersion of parasites among hosts: how general a phenomenon? J Parasitol 75:707-710

Pavlovsky EN (1934) The organism as the environment. Priroda 1:80-91 (in Russian)

Price PW (1980) Evolutionary biology of parasites. Princeton University Press, Princeton, New Jersey, $237 \mathrm{pp}$

Riggs MR, Lemly AD, Esch GW (1987) The growth, biomass and fecundity of Bothriocephalus acheilognathi in a North Carolina cooling reservoir. J Parasitol 73:893-900

Romashov VA (1990) About the population and intrapopulation groups of helminths. In: Roitman VA (ed) Factors which regulate the population processes in helminths. Academy of Science USSR, Pushino, pp. 114-116 (in Russian)
Sergievsky SO, Granovitch AI, Mikhailova NA (1991) The age structure of populations of the gastropod molluscs Littorina saxatilis and L. obtusata in the White Sea. - Trudy Zool Inst, Leningr 233:79-126 (in Russian)

Shigin AA (1978) The role of the free-living stages of trematodes in the community. Parasitologia 12:193-199 (in Russian)

Soprunov FF (1987) The molecular basis of parasitism. Nauka, Moscow, 224 pp (in Russian)

Truc P, Tibayrenc M (1993) Population genetics of Trypanosoma brucei in central Africa: taxonomic and epidemiological significance. Parasitology 106:137-149

Wakelin D (1978) Genetic control of susceptibility and resistance to parasitic infection. Adv in Parasitol 16:219-308

Wassom DL, Dick TA, Arnason N, Strickland D and Grundmann AW (1986) Host genetics: a key factor in regulating the distribution of parasites in natural host populations. J Parasitol 72:334-337

Yablokov AV (1987) Population biology. High School, Moscow, 237 pp (in Russian)

Communicated by H.-D. Franke 